\title{
Anatomical Variations in Condylar Shape and Symmetry: Study of 100 Patients
}

\author{
Dr. Madhumati Singh ${ }^{1}$, Dr. Anindya Chakrabarty ${ }^{2}$ \\ ${ }^{1}$ Professor \& HOD, Dept of Oral \& Maxillofacial Surgery, Rajarajeswari Dental College \& Hospital, RGUHS(No. 14, RAMOHALLI \\ Cross, Mysore Road, Kumbalgodu, Bangalore -560074) \\ ${ }^{2}$ Post Graduate Student, Dept of Oral \& Maxillofacial Surgery, Rajarajeswari Dental College \& Hospital, RGUHS(No. 14, RAMOHALLI \\ Cross, Mysore Road, Kumbalgodu, Bangalore -560074)
}

\begin{abstract}
Anatomical knowledge of the TMJ is one of the foundations of clinical practice, allowing understanding pathological alterations, diagnostic evaluation through the resources of image diagnostics, and choice of appropriate therapeutic paths. Several studies shows there are variations in shape and symmetry of condylar head. The aim of the study was to determine and classify the shape of condyle in different types, relating them to sex and symmetry in a series of orthopantomogram (OPG). The sample included 100-OPG from 14 to 45 years old, 52 males and 48 females. Shapes were classified, antero-posterior width and symmetry verified in this present study. Shapes were classified as rounded, angled, flattened and mixed types in the lateral views. Round type of condyle is more frequent than other varieties. Bilateral symmetry in shape and antero-posterior width is comparatively less common. No as such sex predilection has been found in this present study for variations in shape and symmetry of condyle.
\end{abstract}

Keywords: Temporomandibular joint, Condyle, Orthopantomogram, TMD, neuromascular balance

\section{Introduction}

Anatomical knowledge of the TMJ is one of the foundations of clinical practice, allowing understanding pathological alterations, diagnostic evaluation through the resources of image diagnostics, and choice of appropriate therapeutic paths(1).Among the anatomic characteristics is the presence of an articular disk which guarantees geometrical adaptation between joint faces. This disk of dense connective tissue totally divides the joint cavity in upper compartment, where translational and lateral movements take place through sliding, and lower compartment, where rotational movement takes place(2). These two compartments are morphologically and functionally independent. The bone components of TMJ need careful study and understanding for the simple reason that changes in them imply functional disorders (temporomandibulardisorders - TMD) and pain. The large topographical variability between the condyle and fossa is affected by the shape of the former which suffers changes due to bone remodeling. In the absence of condyle the fossa remains shallow, maintaining the characteristics found in a newborn(3). Several authors have discussed about various types of condyle and fossa shape different times without giving proper classification of condyle and fossa(4). A few authors have examined condyle and fossa morphology in computed tomography (CT) images obtained from patients with TMD and non-patients, describing four fossa shapes(5). Other authors have classified condyle in four types in lateral view - round, angled, flattened and mixed type (6)(fig.1).

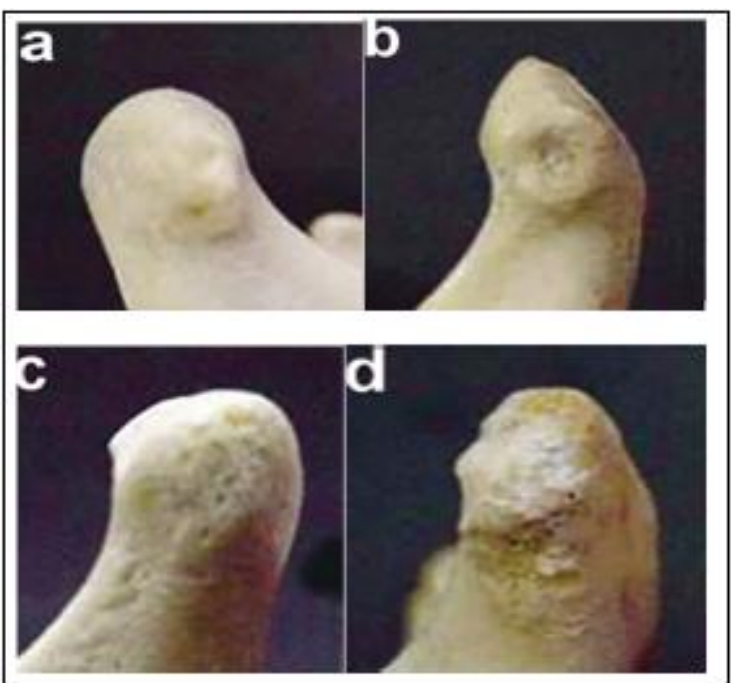

Figure 1: a- round, b- angled, c- flattened, d- mixed

\section{Material and Methods}

The sample consist of 100 human orthopantomogram (14 to 45 year) among which 52 male and 48 female patients radiographs were evaluated. As the orthopantomogram shows the lateral view of the condyle, frequency of various type of condyle, presence of symmetry bilaterally width of condyle by means of antero-posterior dimension was studied.

Data collection include number of various types of condyle, their antero-posterior dimensions and bilateral symmetry of condyle in different patient. 


\section{International Journal of Science and Research (IJSR) \\ ISSN (Online): 2319-7064}

Index Copernicus Value (2013): 6.14 | Impact Factor (2014): 5.611

\section{Results}

In 100 patient 200 condyle were studied thoroughly in orthopantomogram including shape, symmetry and anteroposterior width.

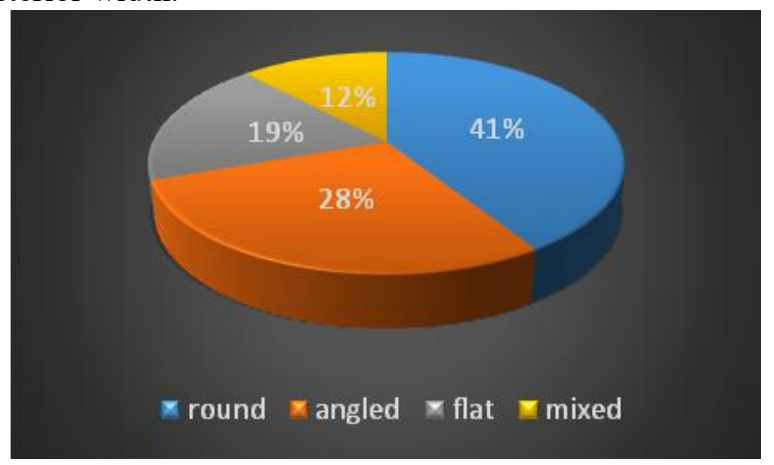

Figure 2: percentage of condylar pattern overall

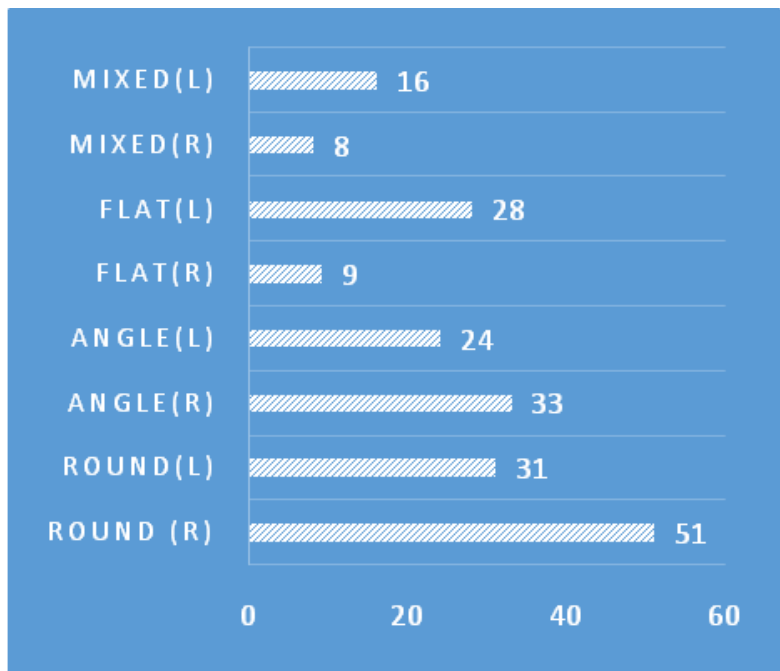

Figure 3: distribution of different shape of condyle

$41 \%$ of total condyle was round in shape (right- $25 \%$ \& left$16 \%$ ), followed by $28 \%$ angled type (right- $17 \%$, left- $12 \%$ ), $19 \%$ was flattened type (right- 5\%, left- 14\%) \& $12 \%$ mixed (right- 4\%, left- 8\%) in shape (fig. 2,3).

The condyle was classified according to shape classification given by Ribeiro, Snaches, Alonso \& Smith, in lateral view - rounded, angled, flattened \& mixed. In orthopantomogram we also found this four type of condyles (fig.4) in anteroposterior view.As per our observation among this varieties rounded variety $(41 \%)$ is more common than other varieties.

In our study most of the patients are within 20 to 35 years of age. We have found that the antero-posterior width vary between $13.03 \mathrm{~mm}$ to $12.32 \mathrm{~mm}$. On comparison of bilateral condylar shape we have found only $35 \%$ of sample shows bilateral symmetry.

Rounded type is more prevalent than other varities and $29 \%$ of sample shown bilateral similarity of antero-posterior width of condyle. And we also found that bilateral symmetrical condyle is more common in female around $66 \%$ (fig. 5,6,7).

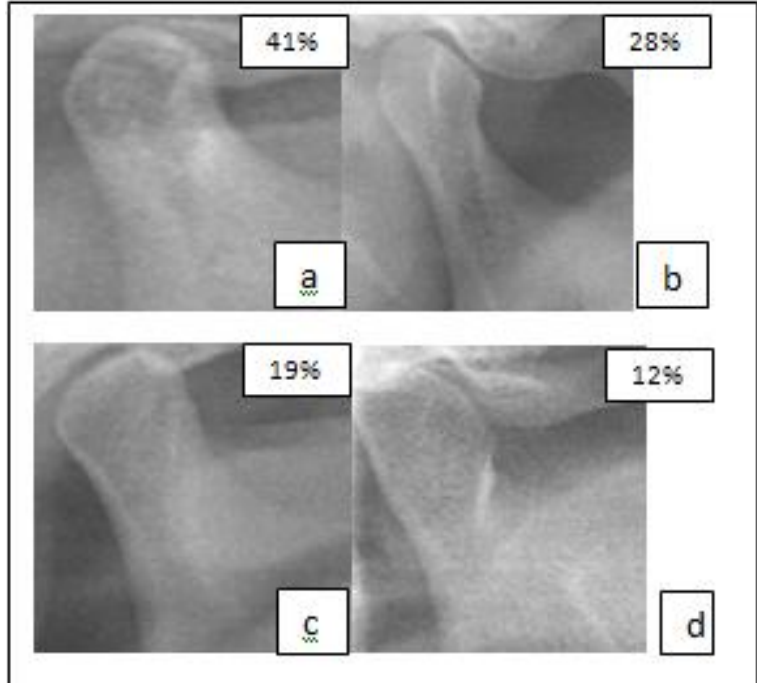

Figure 4: a- rounded, b- angled, c- flattened, d- mixed

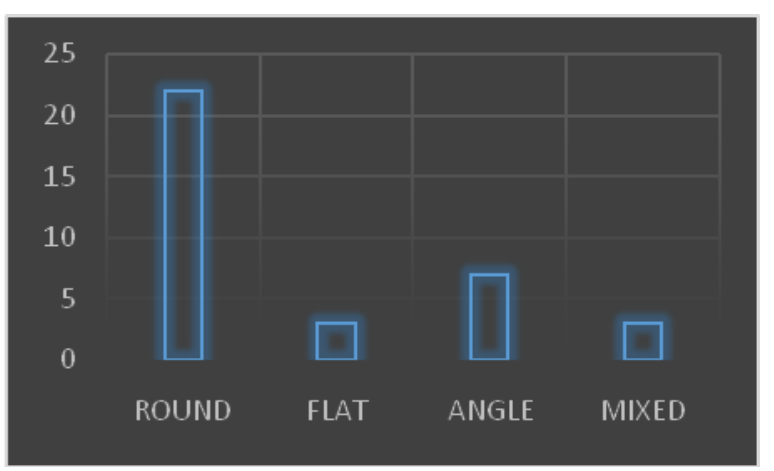

Figure 5: distribution of bilaterally symmetric condyle

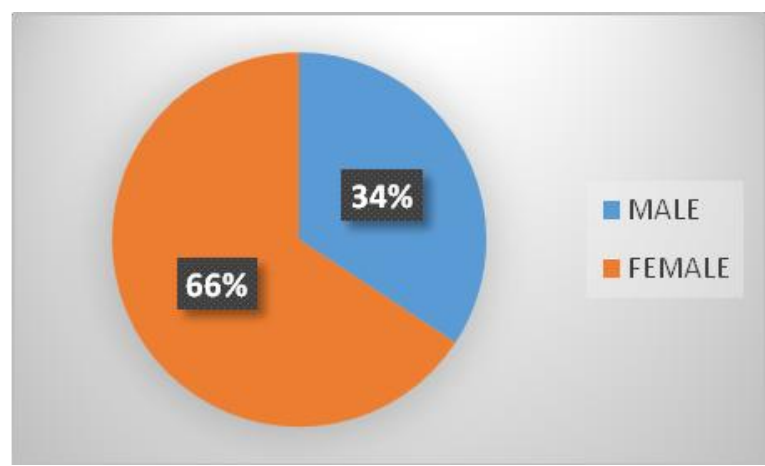

Figure 6: male-female ratio of bilaterally symmetric condyle

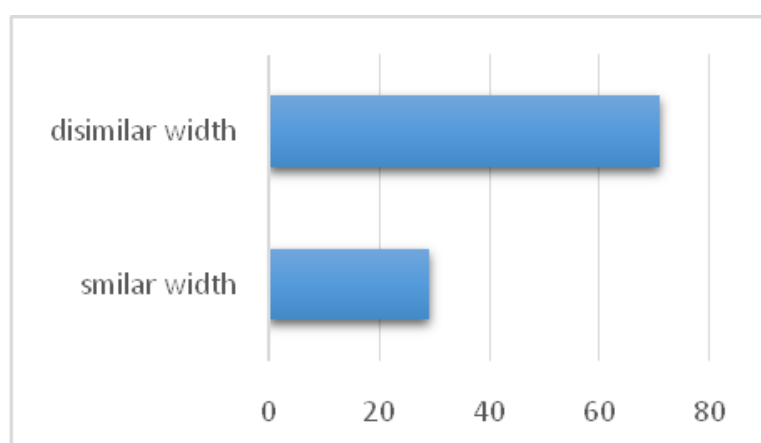

Figure 7: comparison of bilateral symmetrical anteroposterior width of condyle 


\section{International Journal of Science and Research (IJSR) \\ ISSN (Online): 2319-7064}

Index Copernicus Value (2013): 6.14 | Impact Factor (2014): 5.611

\section{Discussion}

Morphological knowledge of the temporomandibular joint (TMJ) is a tool for understandingdevelopment and growth, including phylogenetic aspects and the capacity for bone remodeling characteristic of the skeleton(7). Topographic and shape variability found between the condyle and the mandibular fossa during development and in adulthood has stirred a need for research designed to systematize different shapes found.Condyle shape classification has been elaborated and argued(8)(5)(3) for decades by means of antero-posterior, superior and lateral view radiograph.

Shapes were classified by looking at structure in theorthopantomographic records obtained from our records of 100 patients. According to our study distribution of condylar shapes showed greater frequency of the rounded shape inorthopantomogrma (41\%) followed by angled $(28 \%)$, flattened (19\%) and mixed (12\%) type respectively (fig. 4).

Several authors have studied the symmetry and nonsymmetry of the isolatedCondyle in the three views, yielding significant results for both structures in the lateral view, with predominance of non-symmetry for the mixed shape and of symmetry for the rounded shape(9)(10).

We have found that bilateral symmetry of condyle including shape and antero-posterior dimension is comparatively less frequent. We have found only $35 \%$ of total patient with bilateral shape symmetry of condyle whereas in $29 \%$ of total patients shows equal bilateral antero-posterior width of condyle.In this present study we found that bilaterally symmetrical condyle is more frequent in female subjects $(66 \%)$ in compare to male subject.

\section{Conclusion}

Anatomicaland neurophysiological disturbance in condylar architecture lead to altered neuromuscular balance of temporomandibularjoint. Such alteration create temporomandibular dysfunction (TMD) with advancement of age. As per this study round type of condyle is more frequent among other condylar varieties. Most of the temporomandibular joint consist of non-symmetric condyle. Our study consist of more amount of young and middle aged patient with such variation in condylar shape and symmetry. We can conclude that by fabricating some preventive TMJ care plan for such patients with bilateral asymmetric condyle, we can reduce the incidence of temporomandibular dysfunction in future with advancement of age. So that a thorough knowledge of temporomandibular joint anatomy, condylar variations and neurophysiology of joint can guide clinician in fabrication of proper preventive and curative measures for patients with temporomandibular joint problem.

\section{Ethical Approval}

This article does not contain any studies with human participants performed by any of the authors.

\section{Conflict of Interest}

There is no conflict of interest.

\section{References}

[1] Gray, H. and Williams, P. L. \& Bannister, L.Gray's Anatomy:the anatomical basis of medicine and surgery. 38» ed. New york : Charchil Livingstone, 1995.

[2] Rowe N.L, Williams J L.Maxillofaciall Injuries. s.1. : Elsevier.

[3] Pandis, N., Karpac, J. and Trevino, R. \& Williams, B. A radiographic study of condyle position at various depths of cut in dry skulls with axially corrected lateral tomograms. s.l. : Am. J. Orthod. Dentofacial Orthop., 1991, Vol. 100(2).

[4] Moore, K. L. Dalley, A. F.Anatomia orientada para a clinical. Guanabara Koogan : Rio de Janeiro, 2007.

[5] Raustia, A. M. \& Pyhtinen, J. Morphology of the condyles and mandibular fossa as seen by computed tomography. s.1. : J. Prosthet. Dent., 1990, Vol. 63.

[6] Ribeiro E C, Sanches M L, Alonso G A, Smith R L. Shape and Symmetry of Human Condyle and Mandibular Fossa. s.1. : Int. J. Odontostomat, 2015, Vol. 9.

[7] Christiansen, E. L., et al.Computed tomography of the normal temporomandibular joint. s.l. : Scand. J. Dent.Res., 1987, Vol. 95.

[8] Yale, S. H. and Allison, B. D. , Hauptfuehrer, J. D. An epidemiological assessment of mandibular condyle morphology. s.l. : Oral Surg. Oral Med. Oral. Pathol, 1966, Vol. 21.

[9] Matsumoto, M. A. \& Bolognese, A. M. Bone morphology of the temporomandibular joint and its relation to dental. s.1. : Braz. Dent. J., 1995, Vol. 6.

[10] Katsavrias, E. G. \& Dibbets, J. M. The growth of articular eminence height during craniofacial growth period. s.1. : Cranio, 2001, Vol. 19. 